TECHNOLOGY FEATURE

Set your sites on mutants

\title{
Cloning and mutagenesis: tinkering with the order of things
}

\author{
Engineering the replication of target DNA through cloning, or changing its genetic code through mutations, \\ are detail-oriented processes whose foibles can spell disaster. Caitlin Smith looks at some new tools and \\ techniques that may smooth the road to a successful experiment.
}

'Clone the cDNA from the cells under treatment, insert it into our specialized expression vector, and mutate regions $x, y$ and $z$, which we suspect are important for the gene's function'-this list of instructions could have been a 5 -year thesis project 20 years ago. Today, it is the likely weekly program of many laboratory technicians. Cloning and mutagenesis, once revolutionary techniques that launched the molecular biology era, have now become common techniques no longer constrained to molecular biology labs. The evolution of these basic tools has been spurred by developments brought to the market by many companies. With the myriad of kits available, it seems that cloning and mutagenesis are becoming faster and easier every day.

A recent advance that is proving to be highly efficient and relatively simple is recombineering, now a true alternative to conventional cloning. Though originally observed in Escherichia coli, recombineering seems to export quite well to other model organisms. The technique, whose fine mechanisms are still not completely understood, is attracting increasing attention as a potential solution for manipulating the genome of organisms for which there are fewer tools than for E. coli.

Other methods of mutagenesis have also enjoyed increasing popularity with the advent of large-scale projects to generate collections of mutant mouse lines. The International Gene Trap Consortium, for example, as well as other smaller research groups, are focusing on systematically mutating all genes in the mouse using gene trapping (Box 1). Several groups are also focusing on thematic mutagenesis, systematically knocking down all genes related to a given pathway (Box 2).

\section{BOX 1 GENE TRAPPING FOR THE FUTURE}

Gene trapping is a high-throughput approach to making insertional mutations in mammalian genomes. It is useful because gene traps simultaneously inactivate and report the expression of the 'trapped' gene at the insertion site, and provide a DNA tag for the rapid identification of the disrupted gene. Patricia Ruiz Noppinger from the department of vertebrate genomics at the Max Planck Institute for Molecular Genetics, uses gene trapping in her lab in a large-scale generation of mutant embryonic stem cell lines. Noppinger says that "the most challenging aspect of learning to use gene trapping is the analysis and understanding of the mutation created by the gene trap vector, which includes the determination of the exact integration site of the vector at the genomic level." The method is not without limitations. It has been generally assumed that only about $50 \%$ of mouse genes may be mutated using gene trapping, though Noppinger's lab has evidence that trapping can actually reach up to $70-80 \%$ of targetable genes. Additionally, gene trapping leaves behind cassettes that could have unknown and potentially harmful effects on the mutant phenotype.

William Stanford, director of Gene Trap Mutagenesis at the Centre for Modeling Human Disease at the University of Toronto agrees that "one limitation is that many genes have still not been trapped by any of the labs of the International Gene Trap Consortium (IGTC)." The result of the work in labs participating in the IGTC is that more than $60 \%$ of the genome has been trapped. "There are hot spots for trapping and some genes are refractory to gene trapping," explains Stanford. "Thus, some groups have abandoned gene trapping as a mutagenesis tool and are now performing high-throughput gene targeting. Our poly(A) trap resource has a $70 \%$ unique gene trap hit rate, and thus we are still generating new mutations at a high rate. However, we are part of a large consortium, the North American Conditional Mouse Mutagenesis Project, which is using high-throughput gene targeting to generate conditional mutagenesis alleles in 2,000 genes."

As for the future of gene trapping, Stanford believes that it still has promise for cell lines other than mouse embryonic stem cells- such as human embryonic stem cells and other cell lines, or even for use in mutagenesis of other species. There are presently several gene trap screens being performed in non-murine model organisms.

Noppinger believes that using conditional gene trap vectors together with the ability to determine the insertion sites at the genomic level (for example, by splinkerrette PCR) will greatly affect gene trapping in the future. "Moreover, gene trapping cassettes are currently being designed that will allow recombinasemediated cassette exchange. A further aspect we are thinking about is the potential use of gene trap vectors for gene therapy in the human system." 


\section{Copying, cutting and pasting: fast-tracks for the old-fashioned way}

With increasing ease and sophistication, developments of PCR and other tools have put processes that used to be time-consuming for skilled molecular biologists into every scientist's hands. The PCR products of today are more accurate replicates of their templates, and there is a kit for nearly every possible way of cloning a DNA fragment (from transcribed RNA or another source) into a vector with a specialized function.

For a start, PCR has not only made cloning much easier; improvements to the technology also continue to improve cloning accuracy. "Many people do not realize that reverse transcription is a major contributor to errors when cloning a fragment that originated from RNA," says Rachel Formosa, director of amplification and gene expression product marketing at Stratagene. She explains that conventional reverse transcriptases typically have considerably high error rates, introducing errors at frequencies of one per 1,50030,000 nucleotides during cDNA synthesis. In contrast, Stratagene's Accuscript RT, says Formosa, "contains a 3'-to-5' proofreading exonuclease, which reduces errors three- to sixfold during first-strand cDNA synthesis."

For the next step of cloning cDNA or other DNA fragments, improved PCR enzymes have been available for a while that improve cloning accuracy by reducing the error rates during PCR. For example, Stratagene offers its high-fidelity PfuUltra II Fusion HS DNA polymerase, which according to Formosa generates "only 1 error per 2.5 million bases. It is also good for the replication of long targets, up to $19 \mathrm{~kb}$, and requires only 15 seconds per kb extension time." Likewise, Finnzymes offers their Phusion High-Fidelity DNA polymerase and Phusion Hot Start DNA polymerase. "These polymerases have an extremely low error rate, 50-fold lower than that of Thermus aquaticus DNA polymerase and sixfold lower than that of Pyrococcus furiosus DNA polymerase," says Netta Fatal, marketing communications manager at Finnzymes.

In principle, cloning PCR products necessitates some fancy footwork on the part of the researcher. But in practice with the help of a battery of kits, all you need to know is the nature of the ends of your fragments - staggered, blunt and perhaps containing sites for restriction enzymes. 'Old-fashioned' cloning still works, and for years, researchers relied on using cloning methods in which vector and insert were outfitted with restriction sites to make them

\section{BOX 2 NEUROMUTAGENESIS CENTERS}

A few years ago, the US National Institutes of Health funded three projects scattered around the US that had something in common: a focus on neuromutagenesis the mutation of genes involved in the nervous system. The three locations were the Neurogenomics Project at Northwestern University, the Neuroscience Mutagenesis Facility at The Jackson Laboratory, and the Neuromutagenesis Project of the Tennessee Mouse Genome Consortium. Researchers at all three centers generated mutants lines of mice by randomly generated mutations using a chemical known as ethylnitroso-urea (ENU). To facilitate the sharing of results and the distribution of the resulting mutant mice with other scientists, the three groups came together to form a consortium called Neuromice.org, manifest in a common website through which anyone could order mice. Although no longer functioning as a mutant distribution center, the website still acts as a repository for valuable information, and a directory for mutant lines (which can still be ordered from the participating centers).

Dan Goldowitz's group at the Tennessee Mouse Genome Consortium was one of the three cofounders of Neuromice. org. Goldowitz, a professor in the department of anatomy and neurobiology at the University of Tennessee Health Science Center, explains why he chose to focus on neuromutagenesis: "The nervous system is seen as the last biomedical frontier in several respects, and this is never more clear than in trying to understand the role of single genes in complex behaviors." The goal of Goldowitz's Neuromutagenesis Project is to mutagenize the mouse genome and put the mice through sophisticated screens to identify individual pedigrees to demonstrate neurological phenotypes. "The screens are the sorting mechanism," says Goldowitz, "and unless the mutant had an obvious or a gross phenotype like ataxia or failure to thrive, we designed an unbiased statistical cut-off to tell us which lines to pursue. Those that met that statistical criteria were further developed."

Goldowitz says that there are still problems with trying to study mice with complex phenotypes such as drug-seeking behavior:
"First, the behavioral tests for these phenotypes are inherently noisy, and that makes detecting outliers a difficult problem. Second, as the behavioral readout is not clean, the mapping of the mutant gene is a problem that can not be solved by Mendelian methods. Mutants need to be mapped by quantitative trait locus analytic tools. Third, because of the imprecision of this sort of mapping, it is more challenging to identify the gene. And we all need the gene if we want to call this effort a success."

Joseph Takahashi, Howard Hughes Medical Institute investigator at Northwestern University, heads the Northwestern branch of NeuroMice.org. According to Takahashi, the goal of their program is "to use forward genetics to isolate mouse mutants for neural and behavioral phenotypes. The potential for novel gene discovery is high because forward genetics makes no assumptions on the underlying mechanisms involved and therefore completely unsuspected pathways can be uncovered." He cites the circadian clock and obesity mutants as the best examples of the success of this approach.

Making neuromutants is a laborious process. Takahashi describes how they sort mutants and decide which lines to develop. "We produced and screened 10,000 mice per year and screened over 35,000 mice. We screened using highthroughput behavioral screens in which we could automate data acquisition. Each screen had to have a capacity of more than 200 mice per week (to achieve the goal of 10,000 mice screened per year)." The major screens included fear conditioning, locomotor response to cocaine treatment, motor and balance deficits, visual defects, circadian activity rhythms and serum glucose levels (diabetes).

Takahashi says that the most difficult aspect of creating neuromutants is the development of a robust behavioral screen. Although they "have developed new strategies for mapping behavioral mutants to cope with genetic background issues and the variance of phenotypic assays, much more robust behavioral assays need to be developed for screening." 
compatible. But to avoid additional timeconsuming restriction steps, manufacturers have developed kits with neat tricks to exploit the generic fragment ends generated by Taq polymerases or convert them to blunt ends that will clone efficiently.

Many companies offer cloning kits, including Invitrogen, Lucigen, Promega, Epicentre, Clontech and New England BioLabs, among others. A relatively new kid on the block, Real Biotech Corporation (RBC) wants to beat the clock. The company offers their RBC T\&A cloning kit, in which vectors that contain inserts will also have two new restriction sites, so you can tell whether your DNA fragment has been incorporated. They claim that "the combination of [our] RBC ligation kit and HIT Competent Cells allow the whole process to be completed within six minutes," according to Joyce Kuo, international sales manager at RBC. "RBC's HIT Competent Cells is the fastest transformation system worldwide (completed in less than one minute) and can be applied to all common E. coli laboratory strains."

\section{Set your sites on mutants}

Site-directed mutagenesis has become so valuable an experimental tool that several companies now offer kits to streamline the process. Consider Finnzymes' Phusion SiteDirected Mutagenesis kit for making point mutations, insertions or deletions in plasmid DNA. "The use of Phusion Hot Start DNA polymerase in amplification guarantees extreme fidelity and specificity to the reactions," says Fatal. "No special vectors or restriction sites are required, and also large plasmids (up to $10 \mathrm{~kb}$ ) can be used. The mutagenesis protocol comprises only three steps, making it simple and easy to perform." For generating large libraries of mutated proteins, Finnzymes' transposon-based STOP and MGS kits "offer a convenient way to create saturated libraries of mutated proteins in a single reaction with less hands-on time than any other method."

Stratagene also strives for accuracy with a linear amplification method and high-fidelity DNA polymerase included in its QuikChange mutagenesis kits, which can give results in a day. "Our QuikChange kits do not require single-stranded DNA or labor-intensive, difficult-to-perform steps," says Formosa. "Our trusted, proprietary non-PCR-based QuikChange method ensures that only the parent strand is copied during cycling." This line of products includes competent cells, but is also available in other versions depending on researchers' needs. "We even offer a ver-

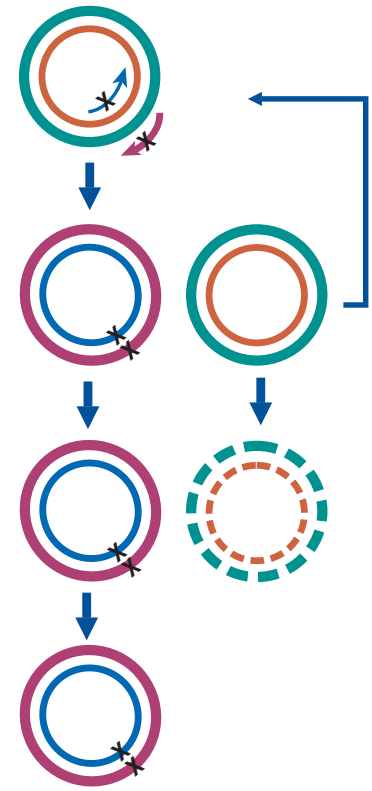

1. Mutant strand synthesis

Perform thermal cycling to:

- denature DNA template

- anneal mutagenic primers containing desired mutation

- extend and incorporate primers with PfuUltra DNA polymerase

2. Dpnl digestion of template Digest parental methylated and hemimethylated DNA with Dpnl

\section{Transformation}

Transform mutated molecule into competent cells for nick repair

The QuikChange II one-day site-directed mutagenesis method. Mutant-strand synthesis is followed by DpnI digestion of the parental DNA template, and transformation of the resulting annealed doublestranded nicked DNA molecules. After transformation, the XL-1 Blue $E$. coli cell repairs nicks in the plasmid. (Courtesy of Stratagene.)

sion made specifically for the electroporation-competent-cell users, as well as a version to introduce multiple point mutations at different sites simultaneously," says Formosa. Other companies offering site-directed mutagenesis kits include Clontech, Invitrogen, Promega and Takara Bio.

\section{Recombineering: how does it work really?} Recombineering, or recombinogenic engineering, is a powerful cloning and mutagenesis technique based on homologous recombination in E. coli. Several systems have been developed that take advantage of phage proteins to efficiently manipulate DNA cloned in bacterial artificial chromosomes (BACs), without the need using restriction enzymes or ligases. The most popular systems are RecET, based on the recE and recT genes encoded by the cryptic RAC prophage that is present in some $E$. coli strains, and lambda Red, based on the homologous recombination system of bacteriophage $\lambda$. The lambda phage Red system is comprised of three functional parts: Red $\alpha$ (or Exo), a 5'-to-3' exonuclease; Red $\beta$ (or Bet or Beta), a protein that binds to ssDNA overhangs generated by Red $\alpha$; and Red $\gamma$ (or Gam), an inhibitor of the major E. coli exonuclease and recombination complex, RecBCD.

Several variations of the recombination system exist in which the three necessary phage proteins Exo, Beta and Gam are expressed and regulated so as to maximize recombination efficiency.

Donald Court, senior investigator at the Center for Cancer Research at the US National Cancer Institute in Frederick, Maryland, USA is one of the pioneers of the technique and an enthusiastic user. He says: "our laboratory uses recombineering for all types of purposes, including gene knock-outs, gene knock-ins, gene fusions, gene point mutations — both directed to a specific nucleotide or randomly generated throughout the gene-gene cloning, plasmid and bacteriophage constructions." A particularly useful application of this technology is the ability to generate targeting vectors for conditional knockouts in mice, quickly and efficiently.

The main focus of Court's lab is to understand the molecular mechanisms of the recombination process, including the contributions of other phage-derived E. coli proteins, with a goal of optimizing the method for genetic engineering": "We have demonstrated that either dsDNA or ssDNA can be used as the substrate for recombineering, and that the replication of the target is a required component. We are aiming to understand what, if any, bacterial proteins are involved in Red recombination."

In the lab of Kenan Murphy, assistant professor in molecular genetics and microbiology at the University of Massachusetts Medical 
School, they are studying two aspects of recombineering technology mechanisms ${ }^{2,3}$. "First, we are examining the biochemistry of $\lambda$ Bet and Exo proteins with dsDNA ends," says Murphy. "We have recently identified a role for $\lambda$ Exo in loading the ssDNA annealing function Bet on to ssDNA. A specific complex of Bet and DNA ends is detected on polyacrylamide gels that is not observed when Bet is simply added to ssDNA after it is generated by Exo."

\section{Manipulating the bad bugs}

Another closely related application lurking on the horizon is vaccine development. Murphy maintains that recombineering could potentially "revolutionize the methods of genetic manipulation in pathogenic microorganisms, leading to faster identification of virulence genes, greater flexibility in the genetic analysis of these genes and the speedy generation of bacterial mutants for vaccine development." Another axis of research in Murphy's lab is looking for Red or RecET-like systems in pathogenic bacteria, to develop recombineering for clinically relevant species.

Such an approach was recently taken by Julia van Kessel in the lab of Graham Hatfull, a professor in the Department of Biological Sciences at the University of Pittsburg. Similar to the recombineering system in E. coli, Hatfull's recombineering system was developed specifically for mycobacteria ${ }^{4}$. "There were good reasons for thinking that a mycobacterial recombineering system would be helpful, since in Mycobacterium tuberculosis, constructing mutants by gene replacement is complicated by a relatively high level of illegitimate recombination," says Hatfull. "We had heard anecdotally that colleagues had attempted to use the E. coli systems in mycobacteria, but with little or no success."

Because the Hatfull lab studies mycobacteriophages (phages that infect mycobacteria), they were able to find mycobacteriophage genes that encode the recombination enzymes that would perform functions analogous to the phage-derived enzymes in E. coli. They identified the rare recombination phage genes, Che $9 c$ genes 60 and 61 ; further characterization revealed that the proteins that they encode were of the correct functional type (that is, an exonuclease and a DNA pairing enzyme). "The primary utility that we have described thus far is the use of mycobacterial recombineering for constructing knockout mutants in M. smegmatis and M. tuberculosis by allelic exchange, although many additional utilities can be imagined."

Work on the mycobacteria recombineering system continues in the Hatfull lab. In general, Hatfull says, it is versatile and simple to use, but "one current limitation is that the recovery of recombinants occurs at somewhat lower frequencies than with the E. coli systems. In and of itself this is not a serious issue for most applications. However, the recombination frequencies are also dependent on the length of the homologous DNA regions, and in practice the number of recombinants recovered with short sequences (that is, $50 \mathrm{bp}$ ) is very low. Thus we typically use $\sim 500$-bp segments of homology, which work well but require somewhat complicated construction of targeting substrates." a Genetic engineering

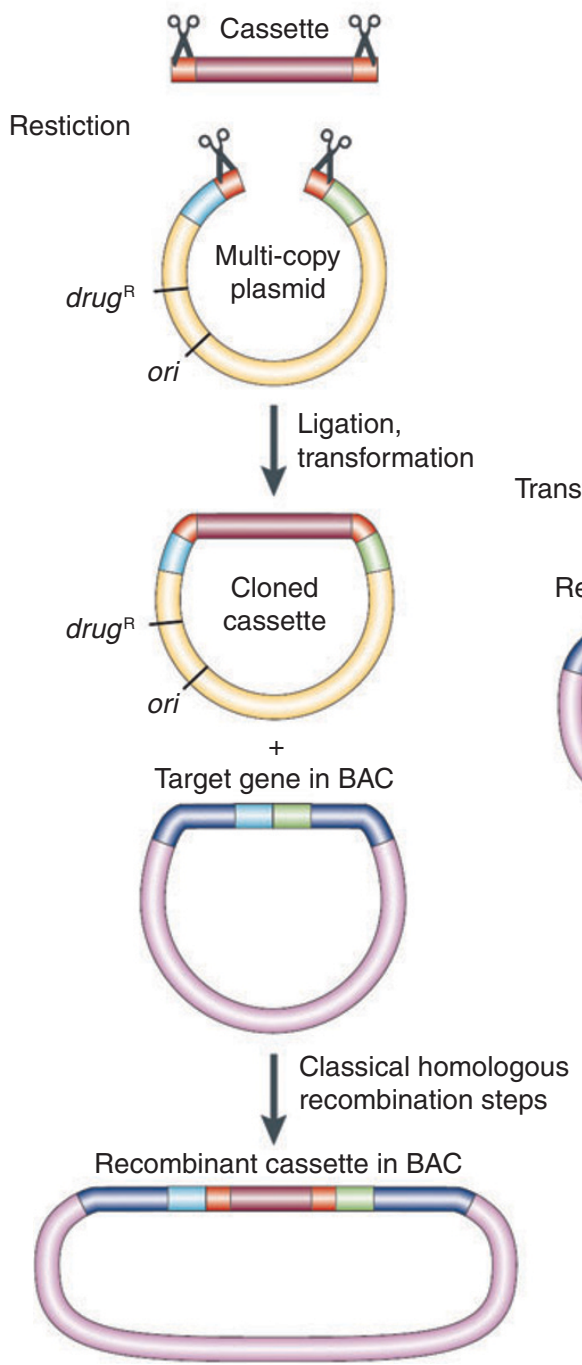

The lab of Don Court has also recently developed a set of plasmid tools to use for recombineering in gram-negative bacteria. The plasmids have been successfully used in Salmonella spp. and are likely to find applications in other gram-negative bacteria.

\section{Recombineering of mice, and flies, and men?}

Recombineering is already used to create mutants with relative ease, and there are more complex applications of this method on the horizon. Tim Townes, a professor in the Department of Biochemistry and Molecular Genetics at the University of Alabama at Birmingham says, "we use recombineering to create all of our constructs for production of knock-in mice. Before recombineering, complicated knock-

b Recombineering

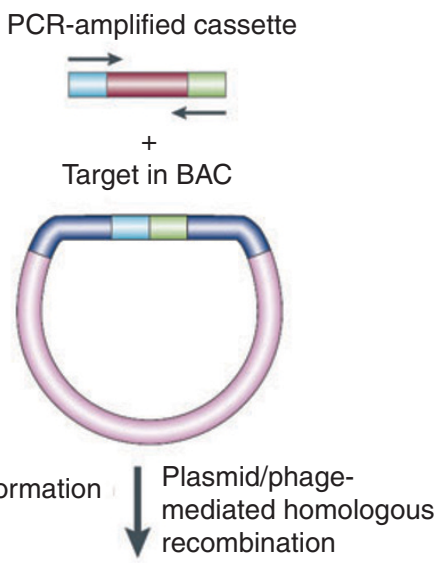

Recombinant cassette in BAC

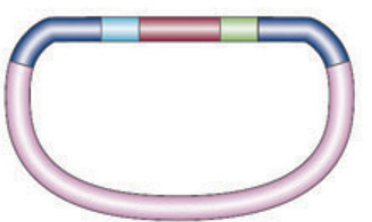

Classical recombinant DNA technology versus recombineering (reprinted from ref. 6 with permission from Nature Reviews Genetics). 


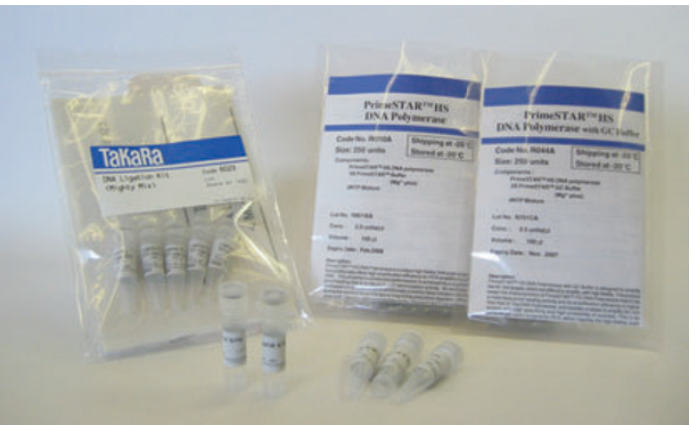

Takara Bio's DNA polymerase and DNA Ligation kit including Mighty Mix. (Courtesy of Takara Bio.)

in plasmids required at least three months to construct. Now constructs can be produced, knock-in [embryonic stem] cells obtained, and knock-in mice derived in 3 months." Likewise, in addition to making simple gene knockouts, Murphy's lab uses recombineering for "BAC manipulation for mouse genetics, chromosomal reporter constructs and genome reductions," says Murphy. "The combination of site-specific recombinases (Flp and Cre) and counterselection markers (sacB and $r p s L)$ creates an almost limitless ability to generate almost any type of chromosomal alteration desired."

Hugo Bellen, a Howard Hughes Medical Institute investigator at the Baylor College of Medicine, and colleagues are presently using recombineering, in conjuction with other methods, to study Drosophila melanogaster genes ${ }^{5}$. They combined recombineering with conditionally amplifiable BAC that supports two crucial features: first, gap-repair of large DNA fragments using recombineering into a single copy plasmid, and second, plasmid copy induction to facilitate the isolation of DNA for Drosophila transgenesis. Moreover, Bellen explains that "the single-copy feature facilitates subsequent recombineering-mediated seamless mutagenesis, as shown by us, and by Colin Dolphin and Ian Hope for Caernohabditis elegans." Bellen's group also incorporated the fC31 system, previously shown to work in Drosophila in vivo to facilitate the integration of large DNA fragments. "There are virtually no limitations concerning the method, since everything is homology based," says Bellen.

Townes predicts that recombineering will be used to insert constructs encoding tandem affinity purification (TAP) tags into most, if not all, endogenous transcription factor genes in the mouse genome, so that transcription factor complexes can be isolated under physiological conditions and defined by mass spectroscopy.
Bellen thinks that tags will be important in the context of high-throughput recombineering. "One important direction will be high-throughput applications of recombineering - for example, to tag each gene of a model organism in a genomic context and visualize its expression in vivo after transgenesis. A variant of high-throughput recombineering has been applied for the modification of hundreds of BAC transgenes in mice and is currently being used for the generation of thousands of gene-targeting constructs."

An eagerly anticipated application of recombineering is gene therapy in mammalian cells. Court explains that the ability to modify the chromosome and do 'gene therapy' in bacteria has progressed rapidly in the last few years. His group's studies with the $\lambda$ Red recombination functions have been critical for this advance. According to Court, efficient gene therapy in mammalian cells using recombination based on the Red functions is a real possibility. "Mammalian viruses like HSV," he explains, "use the same Red-like recombination functions as phage $\lambda$ and should be adaptable for the same purpose."

\section{Do try this at home}

But the use of recombineering is not limited to the few labs that have specialized in its study and its applications to new systems. Murphy says that the challenges of using recombineering as a method are certainly not mechanical. "If you can perform a basic PCR and punch a button on the electroporator, you can do recombineering," he says. But that is not to say that experiments are always successful. "Sometimes we find recombineering not working optimally, and it is usually because our cells are not highly electrocompetent, a drug marker is not expressed well because of chromosomal context effects, the drug concentration being used is too high, or that you're targeting a region of the chromosome that may be a cold spot for recombineering."

With these and perhaps other unknown pitfalls to navigate, some find it useful to rely on the dependability of a kit for alreadytested reagents and procedures. So far, only one company, Gene Bridges, offers kits for researchers doing recombineering. The seven types of kits they offer include the construction of targeting vectors to create transgenic mice (such as conditional knockouts, knock-ins, single-base pair alterations); optimization of E. coli production strains; viral engineering, such as by making point mutations; and RNAi rescue analysis by BAC transgenesis, considered by many to be an important control for specificity in RNAi experiments.

Based on the Red/recET recombination system, each of the kits also contains controls to verify each step in the experiment. "Recombineering as a method is not difficult to apply," says Harald Kranz, head of scientific services at Gene Bridges. "Nevertheless, we realized that it is very important to give customers enough control reactions so that they can set up the recombineering technology step by step in their lab and adjust the reaction conditions to the equipment they have. Giving the possibility to control the efficiency of every step during the reaction is very helpful."

SeqWright now offers custom recombineering services if you want to outsource your experiment. Part of its custom subcloning and mutagenesis services, SeqWright can retrieve DNA fragments from large constructs such as BACs, perform mutagenesis of large constructs and create gene-targeting vectors designed for genetically engineered mice.

Whether you would like a simple cloning kit, or whether you are considering recombineering for gene therapy, the latest developments in cloning and mutagenesis will almost certainly help to speed your experiments along accurately and efficiently.

Caitlin Smith is a science writer based in Portland, Oregon, USA (caitlin. smith@comcast.net).

1. Datta, S. et al. Gene 379, 109-115 (2006).

2. Murphy, K.C. \& Campellone, K.G. BMC Mol. Biol. 4, 11-22 (2003).

3. Campellone, K.G. et al. Mol. Microbiol. 63, 14681481 (2007).

4. van Kessel, J.C., Hatfull, G.F. Nat. Methods. 4 147-152 (2007).

5. Venken, K.J. et al. Science 314, 1747-1751 (2006)

6. Copeland, N.G., Jenkins, N.A. \& Court D.L. Nat. Rev. Genet. 2, 769-779 (2001). 
SUPPLIERS GUIDE: COMPANIES PROVIDING CLONING, RECOMBINEERING AND MUTAGENESIS REAGENTS

\section{Company}

AppliChem

Bioline

Bioron

Bio S\&T

BIOzymTC

CinnaGen

Clontech (owned by Takara Bio)

Epicentre Biotechnologies

Fermentas

Finnzymes

GE Healthcare

Gene Bridges

GenHunter

IngentaConnect

Invitrogen

Jena Bioscience

Lucigen

MacConnell Research

MBL International

New England BioLabs

Novagen

Open Biosystems

Origene

Promega

Qiagen

Real Biotech

Roche Applied Science

SeqWright

Sigma-Aldrich

Stratagene

Takara Mirus Bio

Talron

United Bioinformatica Inc.

\section{Web address}

http://www.applichem.com

http://www.bioline.com

http://www.bioron.net

http://www.biost.com

http://www.biozymtc.com

http://www.cinnagen.com

http://www.clontech.com

http://www.epibio.com

http://www.fermentas.com

http://www.finnzymes.com

http://www.gehealthcare.com

http://www.genebridges.com

http://www.genhunter.com

http://www.ingentaconnect.com

http://www.invitrogen.com

http://www.jenabioscience.com

http://www.lucigen.com

http://www.macconnell.com

http://www.mblintl.com

http://www.neb.com

http://www.emdbiosciences.com

http://www.openbiosystems.com

http://www.origene.com

http://www.promega.com

http://www.qiagen.com

http://www.real-biotech.com

http://www.roche-applied-science.com

http://www.seqwright.com

http://www.sigmaalrich.com

http://www.stratagene.com

http://www.takaramirusbio.com

http://www.talron.co.il

http://www.ubi.ca 